THE WORK OF RAPE 
RANA M. JALEEL / 


\section{THE WORK OF RAPE}

DUKE UNIVERSITY PRESS Durham and London 202I 
(C) 202I DUKE UNIVERSITY PRESS All rights reserved

Printed in the United States of America on acid-free paper $\infty$ Designed by Aimee C. Harrison

Typeset in Garamond Premier Pro

by Westchester Publishing Services

Library of Congress Cataloging-in-Publication Data

Names: Jaleel, Rana M., [date] author.

Title: The work of rape / Rana M. Jaleel.

Description: Durham : Duke University Press, 2021. | Includes

bibliographical references and index.

Identifiers: LCCN 2021005486 (print)

LCCN 2021005487 (ebook)

IS BN 9781478013570 (hardcover)

ISBN 9781478014508 (paperback)

ISBN 9781478021797 (ebook)

Subjects: LCSH: Rape as a weapon of war. | Sex crimes-

Rwanda. | Sex crimes-Yugoslavia. | Women and war. | Women in war. | Women-Violence against-Rwanda. | Women-

Violence against-Yugoslavia. | Rape. | International criminal law. BISAC: SOCIAL SCIENCE / Gender Studies | SOCIAL SCIENCE / Ethnic Studies / General

Classification: LCC KZ7162.J35 202I (print) | LCC KZ7162

(ebook) | DDC 341.6/9-dc23

LC record available at https://lccn.loc.gov/2021005486

LC ebook record available at https://lccn.loc.gov/2021005487

Cover art: Walid Raad, Appendix 137_Ioo (detail), 2018.

Archival inkjet print mounted on Sintra, 34 5/8 x 29 I/ 4 in.

Edition of $5,+2$ APs. (C) Walid Raad. Courtesy Paula Cooper

Gallery, New York.

Publication of this book is supported by Duke University Press's

Scholars of Color First Book Fund. 